\title{
Atemwegs- und Lungenerkrankungen durch Kühlschmierstoffe
}

\section{Olten \\ M. Korn \\ T. Mensing \\ T. Brüning \\ R. Merget}

\section{Airways and Lung Disease by Metal Working Fluids}

\section{Zusammenfassung}

Der Begriff „Kühlschmierstoff“ (KSS) bezeichnet eine meist aus vielen Einzelstoffen bestehende Flüssigkeit, deren Aufgabe die Kühlung und Schmierung von bohrenden und spanhebenden Werkzeugen in der metallverarbeitenden Industrie ist. KSS setzen sich neben einer Wasser-Öl-Emulsion aus einer Reihe weiterer Komponenten zusammen (z.B. Emulgatoren, Biozide). Sie unterliegen während des Gebrauches einer Änderung ihrer Zusammensetzung durch die Bildung von Reaktionsprodukten sowie durch eine mikrobielle Kontamination mit Bakterien und Pilzen. Ziel der vorliegenden Übersicht war es, die Literaturdaten zu Dosis-Wirkungs-Beziehungen hinsichtlich pulmonaler Erkrankungen unter besonderer Berücksichtigung der Belastung mit biologischen Substanzen zusammenzustellen. Die Mehrzahl der Untersuchungen beschreibt obstruktive Atemwegserkrankungen aufgrund irritativer Einflüsse der KSS. Soforttypallergien auf KSS-Bestandteile oder interstitielle Lungenerkrankungen im Sinne einer exogen allergischen Alveolitis können als Raritäten bezeichnet werden. Grundsätzlich weisen die bisherigen Untersuchungen auf schädigende Atemwegseffekte einer KSS-Exposition hin, es bestehen aber noch Defizite hinsichtlich der Definition der Dosis-Wirkungs-Beziehungen. Die Frage, inwieweit Atemwegserkrankungen bei KSS-Exponierten auf eine mikrobielle Kontamination mit Schimmelpilzen und/oder auf eine Endotoxinbelastung zurückgehen, kann bisher nicht hinreichend beantwortet werden. Es bestehen aber schwache Hinweise darauf, dass biologische Stoffe in KSS die wesentlichen schädigenden Substanzen darstellen.

\section{Abstract}

Metal working fluids (MWF) are used in the manufacturing process to cool and lubricate machined parts. MWF contain the coolant, mostly water-oil emulsions with soluble semi-synthetic or synthetic oils, but also emulsifiers and additives. Reaction products and both bacterial and fungal contamination occur during use. In the present review we listed literature data of dose-effect-relationships with a focus on biological substances. Most studies describe obstructive airways disease due to irritant effects of MWF. Immunoglobulin E mediated airways disease or hypersensitivity pneumonitis have rarely been described. There is no doubt that MWF exert adverse effects on human airways, but there is few data concerning dose-effect relationships. The role of bacterial and fungal contamination or endotoxin exposure cannot be defined by the available information. However, there is some evidence that biological components in MWF may represent the most hazardous substances. 
Entsprechend der deutschen Nomenklatur [1,2] werden KSS eingeteilt in nichtwassermischbare KSS (reine Schneidöle), wassermischbare KSS-Konzentrate (Öl-Wasser-Emulsionen), die ihrerseits unterteilt werden in KSS-Emulsionen, KSS-Lösungen und wassergemischte KSS, eine aus wassermischbaren KSS und Wasser hergestellte gebrauchsfertige Mischung (Wasser + Öl-Emulsion). Die Basis für nichtwassermischbare KSS und für KSS-Konzentrate ist in der Regel ein Mineralöl. Die Mineralölanteile der nichtwassermischbaren KSS setzen sich zusammen aus Paraffinen, Zykloparaffinen und Aromaten [3]. KSS-Lösungen besitzen meistens eine synthetische Grundlage. Die amerikanische Einteilung [4] sieht folgende Kategorisierung der KSS vor: Schneidöle $\cong$ nichtwassermischbare KSS (Mineralöl; engl.: straight oils), lösliche Öle $\cong$ wassermischbare KSS (Wasser + Öl-Emulsion; engl.: soluble oils) und synthetische KSS-Lösungen (ölfreie Chemikalien in wässriger Lösung; engl.: synthetic fluids).

Wesentliche Aufgabe der KSS (Primäranforderung) ist es, durch Schmierung die Reibung zwischen Werkzeug und Werkstück zu verringern und die entstandene Wärme (Kühlung) und Späne abzuführen. Zu den Sekundäranforderungen zählen u.a. Lagerstabilität, Korrosionsschutz, Verbesserung des Schaum- und Umweltverhaltens. Um diese Effekte zu erreichen, enthalten KSS so genannte Additive (z.B. schmierfilmbildende Stoffe, Korrosionsschutzzusätze, Schaumverhinderer, Emulgatoren, Konservierungsmittel, Biozide, Geruchsstoffe etc.). Diese erfüllen Anforderungen bezüglich der Druckaufnahme zwischen Werkstück und Werkzeug, Rostschutz, Verringerung von Ölnebeln, Schutz vor Bakterien und Pilzen, Emulgierbarkeit, Entschäumung sowie der Verträglichkeit mit Kunststoffen und Metallen. Der Anteil der Additive beträgt in wassermischbaren KSS maximal 1-2\% und in nichtwassermischbaren KSS bis zu 10\% [5].

Während des Gebrauchs unterliegen die KSS thermischen Veränderungen, einer Verunreinigung durch Schmutz- und Spanpartikel sowie einer mikrobiellen Besiedelung [6]. Vor allem bei Standzeiten von mehr als sechs Monaten können KSS durch chemische, physikalische und biologische Einflüsse verändert werden [1], demzufolge sind Sekundärprodukte zu berücksichtigen. Hieraus ergibt sich die Zusammensetzung der KSS aus Primärstoffen und Sekundärstoffen. Primärstoffe sind Basisstoffe (z.B. Wasser, Mineralöle, synthetische Flüssigkeiten), Zusätze (Additive) und Begleitstoffe, die sich bei der Synthese der Einzelbestandteile als Nebenprodukte bilden. Sekundärstoffe sind Reaktionsprodukte, wie z. B. Nitrosamine, polyzyklische aromatische Kohlenwasserstoffe (PAK), Metalle, Metallsalze, Fremdstoffe (diese werden beim Gebrauch von KSS von außen zugeführt, z.B. durch Metallabrieb, Reiniger, Korrosionsmittel) und Mikroorganismen (Bakterien, Pilze), die insbesondere in wassermischbaren KSS vorkommen [7]. Dabei ist die Besiedlung der KSS mit einer Mischflora (aerobe bis fakultativ anaerobe gramnegative Bakterien) „Wegbereiter“ für nachfolgende Kontaminationen mit kritischen Mikroorganismen wie Anaerobiern, Hefen und Fadenpilzen. Es existiert aber keine typische Leitkeimflora der KSS, vielmehr unterliegt die Artenzusammensetzung einem stetigen Wandel. Reinkulturen sind in KSS nicht überlebensfähig [7].
Weyandt [8] führte eine Simulation von KSS-Kreisläufen mit bakterieller Populationsanalyse durch, bei der sich auch unter praktisch identischen Bedingungen in Parallelansätzen unterschiedliche Organismengesellschaften mit starker qualitativer Veränderung während der Betriebsdauer entwickelten. Typische Leit- oder Identifikationsorganismen ließen sich auch hier nicht ermitteln. Während der Verwendung von KSS sind regelmäßige Kontrollen hinsichtlich der Gebrauchskonzentrationen, des $\mathrm{pH}$ Wertes, der Zahl der Mikroorganismen sowie des Nitrit- und Nitratgehaltes erforderlich. An Pflegemaßnahmen sind zu nennen: Entfernung von festen und flüssigen Verunreinigungen, Zugabe von Bioziden und Begrenzung der Mikroorganismen auf $10^{6} \mathrm{Kei}-$ $\mathrm{me} / \mathrm{ml}$ und $10^{4} \mathrm{Pilze} / \mathrm{ml}$ [2]. Hinsichtlich der Endotoxinkonzentration am Arbeitsplatz (8-Stunden-Schichtmittelwert) wird in den Niederlanden ein Luftgrenzwert von $50 \mathrm{EU} / \mathrm{m}^{3}\left(5 \mathrm{ng} / \mathrm{m}^{3}\right)$ empfohlen [9]. Diese Empfehlung beruht auf einem gesundheitsbasierten Expositionslimit. Ausgangspunkt des Wertes ist der sog. „no effect level“ (NOEL) von $90 \mathrm{EU} / \mathrm{m}^{3}$, der in einer Studie mit asymptomatischen Personen, die gegen Endotoxin-kontaminierten Baumwollstaub exponiert waren, ermittelt wurde. In den von der DECOS [9] vorgeschlagenen Grenzwert ist ein so genannter Sicherheitsfaktor von etwa 2 eingerechnet, der auch Effekte bei geringeren Endotoxinkonzentrationen und auch chronische Expositionsbedingungen in anderen Personengruppen berücksichtigt. Darüber hinaus muss angemerkt werden, dass in anderen Studien die Angaben hinsichtlich eines NOEL zwischen 90 und $1800 \mathrm{EU} / \mathrm{m}^{3}$ schwanken, so dass weitere Untersuchungen erforderlich sind. Vorläufig dient die DECOS-Empfehlung in Deutschland als Orientierung.

Bei der Verwendung von KSS werden entsprechende Dämpfe und Aerosole freigesetzt, die alveolengängig sind [10]. Tierexperimentell ist eine Zellmembranschädigung und Störung der Surfactant-Funktion durch oberflächenaktive Substanzen in wassermischbaren KSS belegt. Eine derartige Wirkung des Mineralölanteils in KSS unter arbeitsüblichen Bedingungen wurde infrage gestellt [5]. Stalder et al. [11] dokumentierten in einer älteren tierexperimentellen Untersuchung zur Erfassung und Quantifizierung einer Zellschädigung durch Konservierungsmittel in KSS (Pentachlorphenol, Hexachlorophen, 4-Chlor-3-Methylphenol) eine besondere Zytotoxizität für Hexachlorophen. Insofern sind Schädigungen der Atemwege und Lungen durch Primär- und Sekundärstoffe plausibel. Ziel dieser Übersicht war es, die tierexperimentellen und Humandaten zusammenzutragen und hinsichtlich der Effekte auf das Zielorgan Lunge/Atemwege beruflich exponierter Personen zu bewerten. Auf Hautkrankheiten soll an dieser Stelle ebenso wie auf die kanzerogene Wirkung von KSS bzw. deren Bestandteile nicht eingegangen werden.

\section{Methodik}

Wir recherchierten in den Datenbanken Medline und Toxline der National Institutes of Health (NIH), USA (http://toxnet.nlm.nih.gov) unter den Begriffen „lubricants“, „metal working fluids“, „coolants“, und „machinist“ jeweils mit der Verknüpfung „airways“, „bronchitis“, „asthma“, „lung“ und „pneumonitis“. Dabei zeigten sich insgesamt 49 Treffer, davon wurden 12 für geeignet gehalten. Elf weitere Arbeiten, die in Medline gelistet waren, wurden aus den Referenzen dieser Literaturstellen re- 
cherchiert. Zusätzlich sind 12 deutsche Arbeiten, die nicht in Medline gelistet waren, aus unserer Institutsliteraturdatenbank eingeschlossen. Beruflich bedingte Hauterkrankungen durch KSS stellen ein wesentlich häufigeres Problem als Atemwegserkrankungen dar [1].

\section{Tierexperimentelle Untersuchungen (Tab.1)}

Stalder et al. [11] wiesen in Inhalationsversuchen bzw. nach intratrachealer Injektion mit Bohr- und Schneidölen an Ratten eine Reizung des zellulären Lungenabwehrsystems sowie eine Änderung der quantitativen Zusammensetzung des Lungen-Surfactant nach. Dalbey et al. [12] führten Langzeitexpositionstestungen (6 Stunden täglich, 5 Tage pro Woche über 4 Wochen) mit frischen Öl-Aerosolen an Ratten mit Konzentrationen von $0,5,210$ und $1000 \mathrm{mg} / \mathrm{m}^{3}$ durch und fanden leichte Entzündungsreaktionen der Lunge und der entsprechenden Lymphknoten im Sinne einer Akkumulation von Alveolarmakrophagen, einer Neutrophileninfiltration sowie einer Verdickung der Alveolarmembran. Nach Einschätzung der Autoren weisen diese Befunde auf einen niedrigen Toxizitätsgrad der Öl-Aerosole hin. Zur Abklärung der irritativen Eigenschaften führten Schaper et al. [13] bei Mäusen inhalative Expositionstests mit zehn KSS bei Konzentrationen von 20 bis $2000 \mathrm{mg} / \mathrm{m}^{3}$ durch. Dabei wurden Atemwegsirritationen mit keinerlei bis geringen Lungenfunktionseinschränkungen für sämtliche KSS festgestellt, wobei die Analyse der Dosis-Wirkungsbeziehung ein besonderes Irritationspotenzial synthetischer und wasserlöslicher KSS im Vergleich zu Schneidölen ergab. Thorne et al. [14] wiesen bei Meerschweinchen dosisabhängige Lungenfunktionseinschränkungen nach inhalativen Expositionstests mit gebrauchten KSS bzw. KSS-Bioziden (Formaldehyd, Methylisothiazolin), nicht aber nach Exposition mit ungenutzten Lösungen nach. Der Endotoxingehalt gebrauchter KSS lag bei 280 bis $1,7 \times 10^{5} \mathrm{EU} / \mathrm{ml}$. In der bronchoalveolären Lavage (BAL) Endotoxin-sensitiver Mäuse bestanden postexpositionell mit gebrauchten KSS Veränderungen des Zellverteilungsmusters im Sinne einer akuten Entzündungsreaktion, welche nach Testung mit ungebrauchten KSS bzw. bei Endotoxin-resistenten Mäusen nicht zu beobachten war. Nach Einschätzung der Autoren sind Endotoxine gebrauchter KSS möglicherweise für die postexpositionelle Entzündungsreaktion der Atemwege von besonderer Bedeutung. Marek et al. [15] und Mensing et al. [16] wiesen in ihren Untersuchungen an Kaninchen eine zunehmende bronchiale Hyperreaktivität als Folge übergrenzwertiger Exposition mit wassermischbaren und nicht-wassermischbaren KSS (bis $50 \mathrm{mg} / \mathrm{m}^{3}$ bzw. bei $220 \mathrm{mg} / \mathrm{m}^{3}$ ) nach. Die Autoren werteten diese Ergebnisse im Sinne einer möglichen Risikoerhöhung für die Entstehung einer obstruktiven Atemwegserkrankung bei beruflicher Exposition mit den untersuchten KSS.

Die tierexperimentellen Studien lassen den Schluss zu, dass die inhalative Belastung v.a. durch synthetische KSS entzündliche Veränderungen der Atemwege verursachen kann, wobei insbesondere der Endotoxingehalt gebrauchter KSS als mögliche Ursache dieser Veränderungen zu berücksichtigen ist. Das atemwegstoxische Potenzial von Ölaerosolen wird als vergleichsweise geringer eingestuft.
Humandaten: Dosis-Wirkungs-Beziehungen hinsichtlich Atemwegserkrankungen (Tab. 2)

Zur Frage akuter oder chronischer Atemwegseffekte durch die Exposition mit KSS wurde eine Reihe von Quer-, Cross-shiftund wenige Längsschnittuntersuchungen durchgeführt (Tab. 2).

Kriebel et al. [17] und Kennedy et al. [18] führten Querschnittuntersuchungen mit arbeitsbezogenen Lungenfunktionsmessungen an KSS-exponierten Zerspanungsarbeitern und unbelasteten Kontrollpersonen sowie parallel dazu Messungen der Luftkonzentration von inhalierbaren KSS-Aerosolen, kultivierbaren Bakterien und Endotoxinen durch. Sie wiesen höhere Konzentrationen dieser Komponenten in den Zerspanungsabteilungen im Vergleich zu KSS-freien Arbeitsbereichen nach. Ferner stellten sie arbeitsbezogene Lungenfunktionseinschränkungen bei KSSExponierten ab Luftkonzentrationen von $0,15 \mathrm{mg} / \mathrm{m}^{3}$ KSS-Aerosol fest. Bei langjährig KSS-Exponierten fand sich eine erhöhte Prävalenz chronischer Atemwegsbeschwerden und eine Einschränkung der Basislungenfunktion.

Verschiedene Autoren [4,19-22] stellten bei KSS-exponierten Personen ebenfalls eine erhöhte Prävalenz arbeitsbezogener Atemwegsbeschwerden im Vergleich zu Kontrollpersonen fest. Greaves et al. [4] nahmen eine Differenzierung zwischen KSS (Schneidöle, synthetische KSS) vor und beschrieben in diesem Zusammenhang eine besondere Toxizität synthetischer KSS, während Sprince et al. [19] ohne Spezifizierung nach KSS-Typen eine Dosis-Wirkungs-Beziehung zwischen tätigkeitsbezogenen Atemwegsbeschwerden und der Gesamtaerosolkonzentration bzw. der Bakterien- und Schimmelpilzbelastung ermittelten. Hinsichtlich der Endotoxinexposition lag ein entsprechender $\mathrm{Zu}-$ sammenhang nicht vor. Massin et al. [22] dokumentierten bei Exponierten eine erhöhte Anzahl von Personen mit bronchialer Hyperreaktivität.

Einige Autoren [23 - 25] schildern Kasuistiken von Personen mit arbeitsbezogenem Asthma, wobei arbeitsbezogene Peak-FlowMessungen bzw. inhalative Provokationstests mit gebrauchten und ungebrauchten KSS unter Laborbedingungen eine signifikante Bronchialobstruktion zeigten. Als Auslöser werden sowohl das in KSS enthaltene Pinienöl als auch die Verunreinigung mit Schimmelpilzen angesehen.

Demgegenüber wiesen andere Autoren keine Atemwegseffekte im Zusammenhang mit einer beruflichen KSS-Exposition nach. In den Querschnittuntersuchungen von Stork et al. $[5,26]$ zur Frage der Häufigkeit einer chronischen Bronchitis bzw. einer akuten arbeitsbezogenen Bronchialobstruktion bei Personen mit inhalativer Schneidölaerosol- bzw. KSS-Exposition ergaben sich keine Hinweise einer schädigenden Wirkung auf den Respirationstrakt, wenngleich ca. 35\% der Exponierten über eine subjektive Belästigung klagten [27].

Es finden sich nur wenige Längsschnittuntersuchungen zur Frage von Atemwegseffekten als Folge einer KSS-Exposition: Besonders hervorzuheben ist die Studie von Kennedy et al. [28] an KSSexponierten Berufsanfängern mit Untersuchungen zu Beginn und nach zweijähriger Lehrzeit zur Erfassung der Effekte einer KSS-Exposition in Bezug auf Atemwegserkrankungen. Parallel 
Tab. 1 Tierexperimentelle Untersuchungen

\begin{tabular}{|c|c|c|c|c|c|c|c|}
\hline Studie & Tierspezies & $\begin{array}{l}\text { Messwerte } \\
\text { KSS-Aerosol }\end{array}$ & $\begin{array}{l}\text { Bakte- } \\
\text { rien }\end{array}$ & Endotoxine & $\begin{array}{l}\text { Ergebnis } \\
\text { Lungenfunktion }\end{array}$ & $\begin{array}{l}\text { Weitere } \\
\text { Untersuchungen }\end{array}$ & Aussagen \\
\hline Mensing et al. [16] & Kaninchen & $\begin{array}{l}\text { Provokationstest mit: } \\
\text { a) wassermischb. KSS bei } \leq 50 \text {, } \\
>50 \mathrm{mg} / \mathrm{m}^{3} \\
\text { b) nicht-wassermischb. KSS bei } 40 \text {, } \\
90,220 \mathrm{mg} / \mathrm{m}^{3} \text { Messverfahren: } \\
\text { siehe Marek et al. [15] }\end{array}$ & k. A. & k. A. & $\begin{array}{l}\text { bronchiale Hyperreaktivität } \uparrow \\
\text { nach Exposition mit wassermischb. } \\
\left(>50 \mathrm{mg} / \mathrm{m}^{3}\right) \text { und nicht-wasser- } \\
\text { mischb. } \mathrm{KSS}\left(220 \mathrm{mg} / \mathrm{m}^{3}\right)\end{array}$ & k. A. & $\begin{array}{l}\text { KSS-Exposition kann zu Atemwegs- } \\
\text { schädigung führen. }\end{array}$ \\
\hline Marek et al. [15] & Kaninchen & $\begin{array}{l}\text { Provokation mit wassermischbaren } \\
\text { KSS mit/ohne Biozide, 0,5, } 5 \\
50 \mathrm{mg} / \mathrm{m}^{3} \text { Messverfahren: Verneb- } \\
\text { lung mit „Bayer-Düse“ }\end{array}$ & k. A. & k. A. & $\begin{array}{l}\text { bronchiale Hyperreaktivität } \uparrow \text { nach } \\
\text { Exposition mit } \geq 5 \mathrm{mg} / \mathrm{m}^{3} \mathrm{KSS} \\
\text { (mit und ohne Biozide) } \\
\text { keine Effekte bei KSS-Exposition } \\
<5 \mathrm{mg} / \mathrm{m}^{3}\end{array}$ & k. A. & $\begin{array}{l}\text { KSS-Exposition kann zu Atemwegs- } \\
\text { schädigung führen. }\end{array}$ \\
\hline Thorne et al. [14] & $\begin{array}{l}\text { Meerschwein } \\
\text { Mäuse: } \\
\text { Endotoxin- } \\
\text { sensitiv/resistent }\end{array}$ & $\begin{array}{l}\text { Provokation mit gebrauchten, unge- } \\
\text { brauchten KSS und KSS-Bioziden } \\
\text { (Formaldehyd, Methylisothiazolin) } \\
\text { Messverfahren: } \\
\text { D: Gravimetrie, Kaskadenimpaktor }\end{array}$ & k. A. & $\begin{array}{l}280-1,7 \times 10^{5} \\
\mathrm{EU} / \mathrm{ml}\end{array}$ & $\begin{array}{l}\text { Lungenfunktionseinschränkungen } \\
\text { nach gebrauchten KSS und } \\
\text { KSS-Bioziden }\end{array}$ & $\begin{array}{l}\text { BAL: dosisabhängige Entzündungs- } \\
\text { reaktion der Lunge nach gebrauch- } \\
\text { ten KSS bzw. bei Endotoxin-sensiti- } \\
\text { ven Mäusen. Keine Entzündungszei- } \\
\text { chen nach ungebrauchten KSS bzw. } \\
\text { bei Endotoxin-resistenten Mäusen }\end{array}$ & $\begin{array}{l}\text { Der Endotoxingehalt gebrauchter } \\
\text { KSS ist möglicherweise Ursache } \\
\text { postexpositioneller Entzündungs- } \\
\text { reaktionen der Lunge. }\end{array}$ \\
\hline Schaper et al. [13] & Mäuse & $\begin{array}{l}\text { Provokation mit } 10 \mathrm{KSS} \\
20 \text { bis } 2000 \mathrm{mg} / \mathrm{m}^{3} \text { über je } 3 \mathrm{~h} \\
\text { Messverfahren: } \\
\text { PN: Glasfaserfilter } \\
\text { D: Gravimetrie }\end{array}$ & k. A. & k. A. & $\begin{array}{l}\text { Dosis-Wirkungsbeziehung von } \\
\text { Atemfrequenz } \downarrow \text { und KSS-Konz.. } \\
\mathrm{RD}_{50}: 100-100000 \mathrm{mg} / \mathrm{m}^{3}\end{array}$ & $\begin{array}{l}\text { Histopathologie: } \\
24 \text { h nach Exposition Nachweis einer } \\
\text { leichten bis mäßigen interstitiellen } \\
\text { Pneumonitis bzw. Bronchopneumo- } \\
\text { nie }\end{array}$ & $\begin{array}{l}\text { Besonderes Irritationspotenzial } \\
\text { synthetischer u. wasserlöslicher } \\
\text { KSS im Vergleich zu Schneidölen. }\end{array}$ \\
\hline Dalbey et al. [12] & Ratten & $\begin{array}{l}\text { Provokationstestung mit Ölaeroso- } \\
\text { len bei } 50,210,1000 \mathrm{mg} / \mathrm{m}^{3} \\
\text { [6 h tgl., } 5 \text { Tage über } 4 \text { Wochen] } \\
\text { Messverfahren: } \\
\text { D: Gravimetrie }\end{array}$ & k. A. & k. A. & k. A. & $\begin{array}{l}\text { Histologie: } \\
\text { ab } \geq 210 \mathrm{mg} / \mathrm{m}^{3} \text { Akkumulation von } \\
\text { Alveolarmakrophagen und Neutro- } \\
\text { phileninfiltration. Dosis-Wirkungs- } \\
\text { Beziehung. Ab } \geq 1000 \mathrm{mg} / \mathrm{m}^{3} \text { leichte } \\
\text { Verdickung der Alveolarmembran }\end{array}$ & $\begin{array}{l}\text { Leichte Veränderungen bei hoch- } \\
\text { konzentrierter Exposition weisen } \\
\text { auf niedrigen Toxizitätsgrad der } \\
\text { Ölaerosole hin. }\end{array}$ \\
\hline Stalder et al. [11] & Ratten & k. A. & k. A. & k. A. & k. A. & $\begin{array}{l}\text { inhalative Provokation bzw. intratra- } \\
\text { cheale Injektion mit Bohr-, Schneid- } \\
\text { ölen und gebrauchten KSS: Vermeh- } \\
\text { rung und Veränderung des Lungen- } \\
\text { Surfactant }\end{array}$ & $\begin{array}{l}\text { Intratracheale Injektion führt zu } \\
\text { Reizungen des zellulären Abwehr- } \\
\text { systems der Lunge. Nach Inhalation } \\
\text { geringere Reaktion. }\end{array}$ \\
\hline
\end{tabular}

$\uparrow=$ Anstieg, $\downarrow=$ Abfall, BAL $=$ Bronchoalveoläre Lavage, $\mathrm{k} . \mathrm{A} .=$ keine Angaben, $\mathrm{PN}=$ Probenahme, $\mathrm{D}=$ Detektion 
hierzu erfolgten 68 personenbezogene Messungen der KSS-Aerosolkonzentration (Mittelwert $=0,46 \mathrm{mg} / \mathrm{m}^{3}$ ). Die Untersuchungen ergaben in der exponierten Gruppe überhäufig Personen mit einer bronchialen Hyperreaktivität, die mit der Exposition v.a. durch synthetische aber auch wassermischbare und wasserfreie mineralölbasierte (nichtwassermischbare) KSS assoziiert war. Die Autoren schlussfolgern, dass schon bei niedrigen Konzentrationen unterhalb des Grenzwertes der ACGIH ([29]; Grenzwert $5 \mathrm{mg} / \mathrm{m}^{3}$ ) Atemwegseffekte auftreten.

Rosenmann et al. [30] führten eine Längsschnittstudie zur Frage der Inzidenz von Atemwegsbeschwerden bei KSS-exponierten Arbeitern sowie Arbeitsplatzmessungen hinsichtlich der KSS-Belastung durch. Lediglich in einem Betrieb wurden KSS-Konzentrationen $>5 \mathrm{mg} / \mathrm{m}^{3}$ in der Umgebungsluft festgestellt. Trotz der insgesamt niedrigen Belastung berichteten annähernd $20 \%$ der Arbeiter insbesondere mit Exposition durch KSS-Emulsionen bzw. synthetische KSS über arbeitsbezogene Atemwegsbeschwerden.

Der Fall eines KSS-exponierten Zerspanungsarbeiters mit dem Bild einer histologisch gesicherten exogen allergischen Alveolitis wird in der Arbeit von Freemann et al. [31] diskutiert, Fox et al. [32] untersuchten 20 KSS-exponierte Zerspanungsarbeiter mit der klinischen Diagnose einer exogen allergischen Alveolitis im Vergleich zu 51 unbelasteten Kontrollpersonen. Die exogen allergische Alveolitis ist nach Einschätzung der Autoren möglicherweise auf die Kontamination der KSS mit Bakterien und Pilzen zurückzuführen. In beiden Arbeiten konnte unseres Erachtens ein eindeutiger Zusammenhang zwischen der KSS-Exposition mit der Erkrankung nicht ermittelt werden.

Zu vergleichbaren Ergebnissen kommen auch Hodgson et al. [33] und Zacharisen et al. [34] in ihren Untersuchungen von Fällen exogen allergischer Alveolitiden in metallverarbeitenden Betrieben. Ein eindeutiger Ursachenzusammenhang mit definierbaren Organismen war nicht erkennbar.

\section{Zusammenfassende Bewertung der humanen Expositionsstudien}

In der vorliegenden Literaturübersicht wurden Arbeiten des Zeitraumes 1970 bis dato berücksichtigt: Der Großteil der Studien beschreibt eine Häufung arbeitsbezogener Symptome im Sinne einer obstruktiven Atemwegserkrankung bei KSS-exponierten Personen. Es finden sich asthmatische Beschwerden aufgrund irritativer Einflüsse von KSS sowie chronische bronchitische Krankheitsverläufe infolge langjähriger KSS-Exposition. Vier Arbeiten [31-34] berichten über exogen allergische Alveolitiden, welche nach Einschätzung der jeweiligen Autoren auf die KSSBelastung zurückgehen. Hierbei ist die diagnostische Beweisführung teilweise zweifelhaft und die Fallzahlen sind zu gering, als dass sich eine eindeutige Zuordnung treffen ließe.

Einige wenige Autoren untersuchten verschiedene KSS-Typen hinsichtlich ihres Wirkungsprofils an den Atemwegen. Dabei wurde postuliert, dass synthetische KSS im Vergleich zu anderen KSS-Typen die höchste Atemwegstoxizität aufweisen. Hier besteht Forschungsbedarf.
Die Arbeitsplatzluftmessungen ergaben überwiegend KSS-Konzentrationen unterhalb des in Deutschland gültigen Grenzwertes von $10 \mathrm{mg} / \mathrm{m}^{3}$, bei denen aber dennoch expositionsbedingte Atemwegsbeschwerden und Lungenfunktionseinbußen ermittelt wurden. Verschiedene Untersucher dokumentierten akute arbeitsbezogene Lungenfunktionseinschränkungen, bei denen z. T. eine Abhängigkeit von der KSS-Aerosolkonzentration im Sinne einer Dosis-Wirkungs-Beziehung gezeigt werden konnte, d.h. ab einer bestimmten Expositionsquantität (in einigen Arbeiten bei $\geq 0,2 \mathrm{mg} / \mathrm{m}^{3} \mathrm{KSS}$-Aerosol) zeigten sich signifikant häufiger und mit steigender Belastung zunehmende arbeitsbezogene Lungenfunktionseinbußen.

Die Ausführungen in den einzelnen Arbeiten zur Belastungshöhe sind vor dem Hintergrund der eingesetzten Messverfahren zu bewerten (Tab. 2). In Deutschland wird bei der Probennahme mittels einer Pumpe ein definiertes Luftvolumen durch ein Glasfaserfilter und einen nachgeschalteten XAD-2-Polymerharzadsorber gesaugt und das in der Luft enthaltene Mineralölaerosol vom Glasfaserfilter zurückgehalten. Das nachgeschaltete XAD2-Harz adsorbiert durch den Luftstrom wieder desorbierende Anteile der Aerosole und Mineralöldampf. Im Labor werden die Aerosole als auch die adsorbierten Dämpfe mittels Tetrachlorethen eluiert und diese Phasen zur infrarotspektrometrischen Messung vereint. Die quantitative Auswertung erfolgt anhand von Kalibrierkurven des am jeweiligen Arbeitsplatz eingesetzten KSS. Ein repräsentatives Mineralölgemisch als Vergleichsstandard (z.B. BIA-Mischstandard) sollte nur noch in Ausnahmefällen in Ermangelung des eingesetzten KSS zum Einsatz kommen. Darüber hinaus sind Querempfindlichkeiten bei Koexposition mit organischen Lösungsmitteln zu beachten. Die in USA gebräuchlichen Verfahren sind mit der in Deutschland verwendeten Methode nur eingeschränkt vergleichbar. Hier werden die gesammelten Aerosole z.T. gravimetrisch, z.T. infrarot-spektrometrisch erfasst, d.h. die Gasphase in Form der KSS-Dämpfe und die unter der Probennahme wieder desorbierenden Aerosolanteile bleiben unberücksichtigt. Dies bedingt eine zu niedrig bestimmte Konzentration, darüber hinaus erlaubt die Gravimetrie methodenbedingt keine Differenzierung nach KSS-Typen.

Die meisten Arbeiten wurden als Querschnitt- und Cross-shiftStudien durchgeführt. Hier sind die Ergebnisse hinsichtlich der Entstehung arbeitsbezogener Atemwegsbeschwerden und Lungenfunktionseinschränkungen bei KSS-exponierten Arbeitern unter Vorbehalt zu werten, da durch den Healthy Worker-Effekt eine Unterschätzung der Erkrankungsprävalenz resultieren kann. Darüber hinaus sind unseres Erachtens Cross-shift-Untersuchungen technisch anspruchsvoll und für die Frage der Kausalität einer KSS-Exposition für obstruktive Atemwegserkrankungen wenig geeignet. Die wenigen Längsschnittuntersuchungen beschreiben vermehrt Lungenfunktionseinschränkungen und chronische Atemwegsbeschwerden bei Arbeitnehmern mit langjähriger KSS-Exposition im Vergleich zu unbelasteten Kontrollpersonen. Die Ergebnisse dieser Kohortenstudien sind aber ebenfalls nur eingeschränkt verwertbar, da die Zahlen der inzidenten Fälle zu gering waren, um sichere Erkenntnisse hinsichtlich der Quantität und Relevanz der Atemwegseffekte einer KSSExposition abzuleiten. Auch war das Expositionsmonitoring hinsichtlich der Anzahl der Messungen nicht ausreichend. 
Tab. 2 Humanstudien

\begin{tabular}{|c|c|c|c|c|c|c|c|}
\hline Studie & Design & Probanden & $\begin{array}{l}\text { Messwerte } \\
\text { KSS-Aerosol }\end{array}$ & Bakterien & Endotoxine & $\begin{array}{l}\text { Ergebnisse } \\
\text { Lungenfunktion }\end{array}$ & Aussagen \\
\hline Hodgson et al. [33] & $\mathrm{Q}$ & $\begin{array}{l}\text { Mitarbeiter (73) eines metall- } \\
\text { verarbeitenden Betriebes } \\
\text { a) mit KSS-Expo. und EAA (16) } \\
\text { Kontrollpersonen: } \\
\text { b) mit KSS-Expo. (61) } \\
\text { c) ohne KSS-Expo. (51) }\end{array}$ & $\begin{array}{l}\text { a) } 0,07 \mathrm{mg} / \mathrm{m}^{3} \\
\text { b) } 0,17 \mathrm{mg} / \mathrm{m}^{3} \\
\text { c) } 0,02 \mathrm{mg} / \mathrm{m}^{3} \\
\text { (Mittelwert) } \\
\text { Messverfahren: } \\
\text { PN: stationär auf Polyvinylfilter } \\
\text { D: Gravimetrie und } \\
\text { IR-Spektroskopie }\end{array}$ & $\begin{array}{l}388 \mathrm{CFU} / \mathrm{m}^{3} \\
953 \mathrm{CFU} / \mathrm{m}^{3} \\
\text { k. A. } \\
\text { (Median) } \\
\end{array}$ & $\begin{array}{l}3,5 \mathrm{EU} / \mathrm{m}^{3} \\
96,2 \mathrm{EU} / \mathrm{m}^{3} \\
3,45 \mathrm{EU} / \mathrm{m}^{3} \\
\text { (Median) }\end{array}$ & $\begin{array}{l}\text { Veränderungen der Lungen- } \\
\text { funktion vor und nach Schicht } \\
\text { bei EAA : } \\
\triangle \mathrm{FEV}_{1}:-6,3 \% \\
\Delta \mathrm{FVC}:-7,2 \%\end{array}$ & $\begin{array}{l}\text { AW-Beschwerden traten häufiger in } \\
\text { Betrieb a) und b) als in Betrieb } \\
\text { c) aber unabhängig von KSS-, } \\
\text { Endotoxin- oder Bakterien- } \\
\text { Exposition auf. } \\
\text { Eine eindeutige Ursache der EAA } \\
\text { war nicht festzustellen. }\end{array}$ \\
\hline Kennedy et al. [28] & L & $\begin{array}{l}\text { Zerspanungslehrlinge (82) } \\
\text { Kontrollpersonen (157) }\end{array}$ & $\begin{array}{l}<0,7 \mathrm{mg} / \mathrm{m}^{3-}-3,65 \mathrm{mg} / \mathrm{m}^{3} \\
\text { (Mittelwert 0,46 mg/m } \\
\text { Messverfahren: } \\
\text { PN: personenbezogen auf } \\
\text { Zelluloseester-Membranfilter } \\
\text { D: Gravimetrie }\end{array}$ & k. A. & k. A. & $\begin{array}{l}\text { Basis: } \\
\text { FEV }_{1}(\%) ; \mathrm{FVC}(\%) \\
\text { E: 100,2; 103,4 } \\
\text { K: 102,3; 106,1 } \\
\text { Verlauf: } \\
\text { FEV }_{1}(\%) ; \mathrm{FVC}(\%) \\
\text { E: 98,5; } 103 \\
\text { K: 100,8; } 106 \\
\text { Pos. Methacholintest: } \\
\text { Basis: } \\
\text { E: } 4 \text { (5\%), K: } 8 \text { (5\%) } \\
\text { Verlauf: } \\
\text { E: } 8(10 \%), \mathrm{K}: 6(4 \%)\end{array}$ & $\begin{array}{l}\text { Im Verlauf Erstentwicklung } \uparrow \text { bzw. } \\
\text { Zunahme einer BHR bei E im } \\
\text { Vergleich zu K. } \\
\text { Nachweis einer Assoziation der BHR } \\
\text { v. a. mit sKSS. }\end{array}$ \\
\hline Fox et al. [32] & $S$ & $\begin{array}{l}\text { Zerspanungsarbeiter (20) mit EAA } \\
\text { Kontrollpersonen (51) }\end{array}$ & $\begin{array}{l}0,0-2,7 \mathrm{mg} / \mathrm{m}^{3} \\
\text { Mittelwert: } 1,0 \mathrm{mg} / \mathrm{m}^{3} \\
\text { Messverfahren: } \mathrm{k.A} \text {. }\end{array}$ & k.A. & k. A. & k. A. & $\begin{array}{l}\text { E zeigten eine serologische Reaktion } \\
\text { auf gebrauchte KSS. } \\
\text { Als Auslöser einer EAA wird mikro- } \\
\text { bielle Kontamination der KSS } \\
\text { angesehen. }\end{array}$ \\
\hline Zacharisen et al. [34] & Q & $\begin{array}{l}\text { Arbeiter einer Automobilmotor- } \\
\text { herstellung (30) mit Atemwegs- } \\
\text { erkrankungen: } \\
\text { EAA (7) } \\
\text { chron. Bronchitis (6) } \\
\text { Asthma (12) }\end{array}$ & $\begin{array}{l}<5 \mathrm{mg} / \mathrm{m}^{3} \\
\text { Messverfahren: } \\
\text { PN: stationär auf Polytetra- } \\
\text { fluoroethylenfilter } \\
\text { D: Gravimetrie }\end{array}$ & $\begin{array}{l}525-4200 \mathrm{CFU} / \mathrm{m}^{3} \\
\text { Nachweis gramnegativer } \\
\text { Bakterien in KSS }\end{array}$ & k. A. & $\begin{array}{l}\text { EAA: } \\
\text { Lungenfunktion } \downarrow \text {, } \\
\text { Methacholintest neg. } \\
\text { Asthma: } \\
5 \times \mathrm{FEV}_{1}<70 \% \text { Soll. } \\
\text { Methacholintest neg. } \\
\text { Chron. Bronchitis: } \\
\text { Methacholintest neg. }\end{array}$ & $\begin{array}{l}\text { Erste Studie mit Nachweis von } \\
\text { Asthma br., EAA und chron. } \\
\text { Bronchitis bei Mitarbeitern eines } \\
\text { Betriebes. Als Ursache werden ange- } \\
\text { sehen: spezifische sensibilisierende } \\
\text { Substanzen und unspezifische } \\
\text { Irritantien. }\end{array}$ \\
\hline Rosenmann et al. [30] & $\mathrm{Q}, \mathrm{L}$ & $\begin{array}{l}\text { Automobilarbeiter ( } 755) \text { aus } 37 \\
\text { verschiedenen Betrieben }\end{array}$ & $\begin{array}{l}<0,1 \mathrm{mg} / \mathrm{m}^{3-3,57} \mathrm{mg} / \mathrm{m}^{3} \\
1 \text { Betrieb }>5 \mathrm{mg} / \mathrm{m}^{3} \\
\text { Messverfahren: } \mathrm{k} . \mathrm{A} .\end{array}$ & k. A. & k. A. & k. A. & $\begin{array}{l}\mathrm{n}=77 \text { aus Betrieben mit KSS-Exposi- } \\
\text { tion }<5 \mathrm{mg} / \mathrm{m}^{3} \text { entwickelten Asthma } \\
\text { nach Tätigkeitsbeginn. } \\
\text { Arbeiter mit Expos. gegen wmKSS } \\
\text { und sKSS hatten mehr Beschw. als } \\
\text { mit Mö. }\end{array}$ \\
\hline
\end{tabular}

Fortsetzung nächste Seite 
Tab. 2 Fortsetzung

\begin{tabular}{|c|c|c|c|c|c|c|c|}
\hline Studie & Design & Probanden & $\begin{array}{l}\text { Messwerte } \\
\text { KSS-Aerosol }\end{array}$ & Bakterien & Endotoxine & $\begin{array}{l}\text { Ergebnisse } \\
\text { Lungenfunktion }\end{array}$ & Aussagen \\
\hline Sprince et al. [19] & $\mathrm{Q}$ & $\begin{array}{l}\text { Zerspanungsarbeiter (183) } \\
\text { Kontrollpersonen (66) }\end{array}$ & $\begin{array}{l}\text { personenbezogen: } \\
\text { (Mittelwerte) } \\
\text { E: } 0,33 \mathrm{mg} / \mathrm{m}^{3} \\
\text { K: } 0,08 \mathrm{mg} / \mathrm{m}^{3} \\
\text { KSS-bezogen: } \\
\text { wmKSS: } 0,38 \mathrm{mg} / \mathrm{m}^{3} \\
\text { sKSS: } 0,31 \mathrm{mg} / \mathrm{m}^{3} \\
\text { Messverfahren: } \\
\text { PN + D: stationäre kontinuier- } \\
\text { liche Streulichtdetektion } \\
\text { (Infrarot-Nähe) }\end{array}$ & $\begin{array}{l}\mathrm{E}: \leq 9,790 \mathrm{CFU} / \mathrm{m}^{3} \\
\mathrm{~K}: \leq 258 \mathrm{CFU} / \mathrm{m}^{3} \\
\mathrm{E}: 67,0 \mathrm{CFU} / \mathrm{m}^{3} \\
\mathrm{~K}: 7,6 \mathrm{CFU} / \mathrm{m}^{3}\end{array}$ & $\begin{array}{l}\text { E: } 3,1 \mathrm{ng} / \mathrm{m}^{3} \\
\text { K: } 3,1 \mathrm{ng} / \mathrm{m}^{3} \\
\text { E: } 5,4 \mathrm{ng} / \mathrm{m}^{3} \\
\text { K: } 1,9 \mathrm{ng} / \mathrm{m}^{3}\end{array}$ & $\begin{array}{l}\text { kein Unterschied der } \\
\text { Lungenfunktion } \\
\text { (d. h. Basiswerte, Cross-shift- } \\
\text { Messungen und in Abhängig- } \\
\text { keit von KSS-Typen) zwischen } \\
\text { E u. K. }\end{array}$ & $\begin{array}{l}\text { E hatten häufiger Atembeschwerden } \\
\text { Assoziation zwischen Husten u. Aus- } \\
\text { wurf mit der KSS-Aerosol- und Bakte- } \\
\text { rien-Konzentration, trotz niedriger } \\
\text { Exposition. } \\
\text { Kein Zusammenhang zwischen Endo- } \\
\text { toxin-Belastung und Atembeschwer- } \\
\text { den bzw. Lungenfunktion. }\end{array}$ \\
\hline Kriebel et al. [17] & $\mathrm{Q}$ & $\begin{array}{l}\text { Zerspanungsarbeiter (216) } \\
\text { Kontrollpersonen (170) }\end{array}$ & $\begin{array}{l}\mathrm{E}: 0,2 \mathrm{mg} / \mathrm{m}^{3} \\
\mathrm{~K}: \leq 0,08 \mathrm{mg} / \mathrm{m}^{3} \\
\text { Messverfahren: } \\
\text { PN: personenbez. } \\
\text { Polycarbonatfilter } \\
\text { D: Gravimetrie, danach } \\
\text { Splitten für Endotoxin- und } \\
\text { Bakterienanalyse }\end{array}$ & $\begin{array}{l}\mathrm{E}: \leq 135 \mathrm{CFU} / \mathrm{m}^{3} \\
\mathrm{~K}: \leq 29 \mathrm{CFU} / \mathrm{m}^{3}\end{array}$ & $\begin{array}{l}\mathrm{E}: \leq 16 \mathrm{EU} / \mathrm{m}^{3} \\
\mathrm{~K}: \leq 8,6 \mathrm{EU} / \mathrm{m}^{3}\end{array}$ & 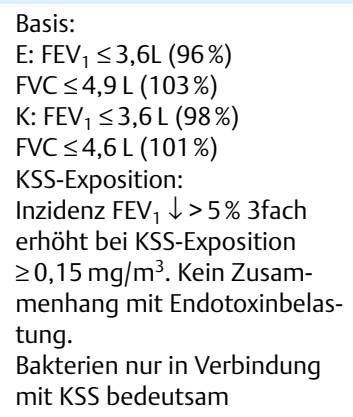 & $\begin{array}{l}\text { Prävalenz } \uparrow \text { chronischer Atem- } \\
\text { beschwerden bei E } \\
\text { (Schneidöle > wmKSS). }\end{array}$ \\
\hline Greaves et al. [4] & Q & $\begin{array}{l}\text { Automobilarbeiter (1.811) } \\
\text { Kontrollpersonen (769) }\end{array}$ & $\begin{array}{l}\text { Schneidöl: } \\
0,43 \pm 0,26 \mathrm{mg} / \mathrm{m}^{3} \\
\text { WmKSS: } 0,55 \pm 0,17 \mathrm{mg} / \mathrm{m}^{3} \\
\text { sKSS: } 0,41 \pm 0,08 \mathrm{mg} / \mathrm{m}^{3} \\
\text { Messverfahren: } \\
\text { PN: personenbez. in 3-stufi- } \\
\text { gem Kaskadenimpaktor } \\
\text { D: k.A. }\end{array}$ & k. A. & k. A. & k. A. & $\begin{array}{l}\text { Assoziation zwischen irritativen } \\
\text { Atembeschwerden und Exposition } \\
\text { v. a. mit sKSS trotz niedrigster Konz. } \\
\text { Aktuelle Belastungen haben größere } \\
\text { Effekte als kumulative Exposition. }\end{array}$ \\
\hline Robins et al. [35] & $\begin{array}{l}\text { L } \\
\text { Cross- } \\
\text { shift } \\
3 \text { Phasen }\end{array}$ & $\begin{array}{l}\text { Zerspanungsarbeiter (83) } \\
\text { Kontrollpersonen (46) }\end{array}$ & $\begin{array}{l}\text { E: MW } 0,56 \mathrm{mg} / \mathrm{m}^{3} \\
\text { K: MW 0,13 mg/m } \\
\text { Messverfahren: } \\
\text { PN: personenbez. auf Memb- } \\
\text { ranfilter aus Polycarbonat im } \\
\text { 2-Stufen-Impaktor } \\
\text { D: k. A. }\end{array}$ & $\begin{array}{l}\text { E: } 3,0 \text { Bakterien } / \mathrm{m}^{3} \\
\text { K: } 0,2 \text { Bakterien } / \mathrm{m}^{3}\end{array}$ & k. A. & $\begin{array}{l}\text { FEV }_{1} \downarrow(\geq 5 \%) \text { (Cross-shift): } \\
\text { E: } 42,9 \% \\
\text { K: } 26,8 \% \\
\text { FVC } \downarrow \text { ( } \geq 5 \%): \\
\text { E: } 41,6 \% \\
\text { K: } 19,5 \% \\
\text { FEV } \downarrow \text { ( }(\geq 10 \%): \\
\text { E: } 16,9 \% \\
\text { K: } 7,3 \% \\
\text { FVC } \downarrow \text { ( } 210 \%): \\
\text { E 6,5\% } \\
\text { K: } 2,4 \%\end{array}$ & $\begin{array}{l}\text { E häufiger chron. und arbeitsbezogene } \\
\text { akute Atembeschwerden als K. } \\
\text { Assoziation zw. Cross-shift Lungen- } \\
\text { funktionsänderungen (v. a. montags) } \\
\text { in Abhängigk. von KSS-Konz. und } \\
\text { Luftbelastung mit Bakterien. }\end{array}$ \\
\hline
\end{tabular}


Tab. 2 Fortsetzung

\begin{tabular}{|c|c|c|c|c|c|c|c|}
\hline Studie & Design & Probanden & $\begin{array}{l}\text { Messwerte } \\
\text { KSS-Aerosol }\end{array}$ & Bakterien & Endotoxine & $\begin{array}{l}\text { Ergebnisse } \\
\text { Lungenfunktion }\end{array}$ & Aussagen \\
\hline $\begin{array}{l}\text { Wild, Ameille [37] } \\
\text { (Neubewertung der } \\
\text { Studie v. Ameille et al. } \\
\text { [38]) }\end{array}$ & - & - & - & - & - & $\begin{array}{l}\text { gegenüber löslichen Ölen E } \\
\text { zeigen eine steilere Dosis- } \\
\text { Wirkungskurve (\%Abfall } \\
\mathrm{FEV}_{1} / \mu \text { mol Methacholin; } \\
\mathrm{p}=0,04 \text { ) }\end{array}$ & $\begin{array}{l}\text { Beide Publikationen zeigen adverse } \\
\text { Effekte auf Atemwege. Neufestset- } \\
\text { zung von Grenzwerten wird } \\
\text { angeregt. }\end{array}$ \\
\hline Massin et al. [33] & $\mathrm{Q}$ & $\begin{array}{l}\text { Metallarbeiter (114) } \\
\text { Kontrollpersonen (55) }\end{array}$ & $\begin{array}{l}\mathrm{CM} 0,65-2,2 \mathrm{mg} / \mathrm{m}^{3} \\
\text { Messverfahren: } \\
\text { PN: stationär auf Glasfilter } \\
\text { D: Gravimetrie nach } \\
\mathrm{CH}_{2} \mathrm{Cl}_{2} \text {-Elution }\end{array}$ & k. A. & k. A. & $\begin{array}{l}\text { pos. Methacholin-Test: } \\
\text { E: } 10(9 \%) \\
\text { K: } 4(8 \%)\end{array}$ & $\begin{array}{l}\text { sign. Prävalenz } \uparrow \text { von Atemwegs- } \\
\text { beschwerden und BHR bei E im Ver- } \\
\text { gleich zu K }\end{array}$ \\
\hline Ameille et al. [37] & $\mathrm{Q}$ & $\begin{array}{l}308 \text { Mitarbeiter einer Autofabrik } \\
40 \text { Schneidöle (S) } \\
51 \text { lösliche Öle (E) } \\
139 \text { S+E (D) } \\
78 \text { Kontrollen }\end{array}$ & $\begin{array}{l}\text { AM: } 2,6 \pm 1,8 \mathrm{mg} / \mathrm{m}^{3} \\
\mathrm{GM}: 2,2 \pm 1,9 \mathrm{mg} / \mathrm{m}^{3} \\
10 \%>5 \mathrm{mg} / \mathrm{m}^{3} \\
\text { Messverfahren: Gravimetrie }\end{array}$ & k. A. & k. A. & $\begin{array}{l}\mathrm{FEV}_{1}, \mathrm{FEF}_{25-75}, \mathrm{~V}_{50}, \mathrm{~V}_{25} \text { bei } \\
\text { Rauchern + S signifikant } \\
\text { erniedrigt }\end{array}$ & $\begin{array}{l}\text { S und D: erhöhte Prävalenzen für } \\
\text { Husten und Schleimbildung } \\
(p=0,048)\end{array}$ \\
\hline Stork et al. [26] & $\mathrm{Q}$ & Zerspanungsarbeiter (34) & $\begin{array}{l}1,2 \pm 0,41 \mathrm{mg} / \mathrm{m}^{3} \\
\text { Messverfahren: } \\
\text { PN: an } 12 \text { verschiedenen } \\
\text { Messstellen im Atembereich } \\
\text { D: Streulichtphotometer }\end{array}$ & k. A. & k.A. & $\begin{array}{l}\mathrm{FEV}_{1}(\mathrm{~L}) \text { vor Schicht 3,99, } \\
\text { nach Schicht } 4,0\end{array}$ & $\begin{array}{l}\text { keine akute expositionsabhängige } \\
\text { Atemwegsobstruktion } \\
\text { arbeitsbez. Beschwerden Folge einer } \\
\text { Schleimhautirritation }\end{array}$ \\
\hline Kennedy et al. [18] & $\mathrm{Q}$ & $\begin{array}{l}\text { Zerspanungsarbeiter (89) } \\
\text { Kontrollpersonen (42) }\end{array}$ & $\begin{array}{l}\text { E: } 0,16-2,03 \mathrm{mg} / \mathrm{m}^{3} \\
\text { K: } 0,07-0,44 \mathrm{mg} / \mathrm{m}^{3} \\
3 \text { Kategorien: } \\
\text { gering: }<0,2 \mathrm{mg} / \mathrm{m}^{3} \\
\text { mittel: } \leq 0,55 \mathrm{mg} / \mathrm{m}^{3} \\
\text { hoch: }>0,55 \mathrm{mg} / \mathrm{m}^{3} \\
\text { Messverfahren: } \\
\text { PN: personenbezogen in } \\
\text { 3-stufigem Kasakaden- } \\
\text { impaktor nach Rubrow } 1987 \\
\text { D: k. A. }\end{array}$ & k. A. & k. A. & $\begin{array}{l}\text { Cross-shift FEV } \downarrow \text { } \downarrow \text { (>5\%) bei } \\
\text { KSS-Exposition }>0,2 \mathrm{mg} / \mathrm{m}^{3} \\
23,6 \% \mathrm{E} \text { u. } 9,5 \% \mathrm{~K} \\
\text { Keine zunehmende Einschrän- } \\
\text { kungen der Lungenfunktion im } \\
\text { Laufe einer Woche in beiden } \\
\text { Gruppen }\end{array}$ & $\begin{array}{l}\text { Nachweis arbeitsbezogener Lungen- } \\
\text { funktionseinschr. gleichen Ausmaßes } \\
\text { zu Beginn und am Ende einer } \\
\text { Arbeitswoche }\end{array}$ \\
\hline Stork et al.[26] & $\mathrm{Q}$ & $\begin{array}{l}\text { Metallarbeiter, Umgang mit } \\
\text { Schneidölen(147) } \\
\text { Kontrollpersonen (172) }\end{array}$ & $\begin{array}{l}0,29-38,5 \mathrm{mg} / \mathrm{m}^{3} \\
\text { Median: } 4,5 \mathrm{mg} / \mathrm{m}^{3} \\
\text { Mittelwert: } 11 \mathrm{mg} / \mathrm{m}^{3} \\
\text { Messverfahren: } \\
\text { PN: k. A. } \\
\text { D: Tyndallometer }\end{array}$ & k. A. & k. A. & k. A. & $\begin{array}{l}\text { keine Hinweise einer AW-schädigen- } \\
\text { den Wirkung von Schneidölen } \\
\text { alle E klagten über subjektive Belästi- } \\
\text { gung durch Ölnebel (ab } 11 \mathrm{mg} / \mathrm{m}^{3} \text { ) }\end{array}$ \\
\hline Järvholm et al.[21] & Q & $\begin{array}{l}\text { Zerspanungsarbeiter (164) } \\
\text { Dreher } \\
\text { Schleifer } \\
\text { Härter } \\
\text { Kontrollpersonen (159) }\end{array}$ & $\begin{array}{l}\text { (Median) } \\
\text { Dreher: } 2,0 \mathrm{mg} / \mathrm{m}^{3} \\
\text { Schleifer: } 3,2 \mathrm{mg} / \mathrm{m}^{3} \\
\text { Härter: } 4,5 \mathrm{mg} / \mathrm{m}^{3} \\
\text { Messverfahren: } \\
\text { PN: Glaswollefilter } \\
\text { D: k.A. }\end{array}$ & k.A. & k.A. & $\begin{array}{l}\text { kein Unterschied der Lungen- } \\
\text { funktion zwischen E und K }\end{array}$ & $\begin{array}{l}\text { E (v. a. Härter) häufiger Atembe- } \\
\text { schwerden (Husten, Auswurf) als K }\end{array}$ \\
\hline
\end{tabular}

Fortsetzung nächste Seite 
Bezüglich der Prävention ist angesichts des zunehmenden Einsatzes gekapselter Maschinen und neuer Schmiertechniken [35] ein Handlungsbedarf hinsichtlich weiterer umfangreicher Studien zu hinterfragen. Die Beurteilung des Kausalzusammenhanges zwischen KSS-Exposition und Atemwegserkrankungen in Berufskrankheiten-Verfahren ist aufgrund der vorliegenden Daten schwierig. Grundsätzlich kann ein Kausalzusammenhang auch bei Einhaltung des in Deutschland etablierten Grenzwertes von $10 \mathrm{mg} / \mathrm{m}^{3}$ keinesfalls ausgeschlossen werden. Zusammenfassend sind Atemwegseffekte einer KSS-Exposition evident, es handelt sich fast ausschließlich um irritative Effekte. Es bestehen aber noch Defizite hinsichtlich der Definition der Dosis-Wirkungs-Beziehungen.

\section{Konzentration biologischer Komponenten und ihre Effekte}

Interstitielle Lungenkrankheiten [31,32] oder Asthma [25] als Folge einer Sensibilisierung auf KSS-Bestandteile (Schimmelpilze) werden nur in Einzelfällen (bei Asthma nur zwei schlecht dokumentierte Kasuistiken) beschrieben. Dosis-Wirkungs-Beziehungen zwischen der Schimmelpilzkonzentration am Arbeitsplatz und Atemwegsbeschwerden wurden bisher nicht systematisch untersucht. Prick-Hauttests mit Schimmelpilzen oder andere Tests zum Nachweis einer Typ-I-Sensibilisierung erfolgten bisher in epidemiologischen Studien nicht.

Es existieren nur wenige Untersuchungen zur Bakterien- und/ oder Endotoxinwirkung durch die Verwendung von KSS für die Entstehung von Atemwegserkrankungen bzw. diesbezügliche Vergleiche zwischen ungebrauchten und gebrauchten KSS. Die Autoren interpretieren in diesen Fällen arbeitsbezogene Atemwegsbeschwerden oder Lungenfunktionseinschränkungen als Folge einer KSS-Belastung bzw. der bakteriellen Kontamination $[17,19,36]$, während für die Exposition mit Endotoxinen keine eindeutigen Assoziationen mit adversen Atemwegseffekten festgestellt wurden $[17,19]$.

In der Literatur finden sich keine Interventionsstudien an Arbeitsplätzen nach verbesserter Wartung der KSS-Kreisläufe, um Kenntnisse über Atemwegseffekte einer KSS-Exposition in Abhängigkeit vom Grad der mikrobiellen Kontamination zu erhalten.

Ob und inwieweit Atemwegserkrankungen bei KSS-exponierten Personen auf eine mikrobielle Kontamination mit Schimmelpilzen und/oder auf eine Endotoxinbelastung in KSS zurückzuführen sind und welche nutzungsbedingten Veränderungen der KSS hierfür eine Rolle spielen, kann derzeit nicht hinreichend beantwortet werden. Allerdings macht der Vergleich der Endotoxinbelastung KSS-exponierter Zerspanungsarbeiter $\left(<10 \mathrm{ng} / \mathrm{m}^{3}\right)$ mit anderen Berufsgruppen (in der Hühnerzucht, Kartoffelbearbeitung und bei Müllarbeitern wurden Werte gemessen, bei denen die Belastung bis zu mehr als das 1000fache beträgt) eine schädigende Wirkung dieser Komponente trotz Hinweisen aus Tierversuchen wenig plausibel. Es fehlen aber weitere auf diese Fragestellung zugeschnittene Studien, anhand welcher die in den bisherigen Untersuchungen gefundenen Hinweise auf einen Kausalzusammenhang zwischen Atemwegserkrankungen und der mikrobiellen Kontamination von KSS ausreichend belegt 
bzw. die offenen Fragen zum Einfluss einer KSS-bedingten Endotoxinexposition auf die Atemwege weiter untersucht werden. Diese Studien sind schwer durchzuführen, da eine Abtrennung der Wirkung biologischer Komponenten von Wirkungen durch die KSS selbst nur durch sehr große Kollektive oder durch Interventionsstudien möglich wäre. Deshalb wäre es unseres Erachtens zunächst sinnvoll zu prüfen, ob bei KSS-Exponierten an gut gewarteten, modernen Anlagen überhaupt Effekte detektierbar sind, bevor man die Untersuchungen auf konkrete Inhaltsstoffe spezifiziert.

\section{Literatur}

${ }^{1}$ Häusser M. Kühlschmiermittel-Bestandteile und ihre gesundheitliche Wirkung. Zbl Arbeitsmedizin 1985; 35: 176-181

2 Sicherer Umgang mit Kühlschmierstoffen. Sicher arbeiten 1993; 3 : $151-158$

${ }^{3}$ Stalder K, Pantke T. Erfassung der Zytotoxizität von Additiven in Kühlschmiermitteln. Verh Dtsch Ges Arbeitsmed Stuttgart: Gentner Verlag, 1985: 439-441

${ }^{4}$ Greaves IA, Eisen EA, Smith TJ, Pothier LJ, Kriebel D, Woskie SR, Kennedy SM, Shalat S, Monson RR. Respiratory health of automobile workers exposed to metal-working fluid aerosols: respiratory symptoms. Am J Ind Med 1997; 32: 450-459

${ }^{5}$ Stork J, Heger M, Nöring R, Thamm H. Inhalative Belastung durch KSSAerosole - arbeitsmedizinische Aspekte. Zbl Arbeitsmed 1995; 45: $228-236$

${ }^{6}$ Hayda E. Wirkung von Kühl- und Schneidmedien auf die Atemwege. Information Arbeitsmedizin und Arbeitsschutz 1989; 2: 67-70

${ }^{7}$ Warfolomeow I. Keimbelastung wassergemischter Kühlschmierstoffe. Die BG 1998; 109: 274-281

8 Weyandt RG. Forschungsprojekt: Kontrolle mikrobiellen Befalls wassergemischter Kühlschmierstoffe. Institut Fresenius Gruppe, 1996

${ }^{9}$ Report of the Dutch Committee on Occupational Standards of the Dutch Health Council. Endotoxins. Health based recommended occupational exposure limit for endotoxins. Minister and State Secretary of social affairs and employment, 1998

${ }^{10}$ Stalder K. Aerosole aus Kühl-Schmiermitteln als arbeitshygienisches Problem. Arbeitsmed Sozialmed Präventivmed 1984; 19: 265-268

${ }^{11}$ Stalder K, Ladany E, Kroll A, Pantke T. Tierexperimentelle Untersuchungen zur Gesundheitsbeeinträchtigung durch Ölnebel. Dortmund: Bundesanstalt für Arbeitsschutz, Forschungsprojekt 625.1, 1985

${ }^{12}$ Dalbey W, Osimitz T, Kommineni C, Roy T, Feutson M, Yang J. Fourweek inhalation exposure of rats to aerosols of three lubricant base oils. J Appl Toxicol 1991; 11: 297-302

${ }^{13}$ Schaper M, Detwiler K. Evaluation of the acute respiratory effects aerolized machining fluids in mice. Fundam Appl Toxicol 1991; 16: 309-319

${ }^{14}$ Thorne PS, DeKoster JA. Pulmonary effects of machining fluids in guinea pigs and mice. Am Ind Hyg Ass J 1996; 75: 1168-1172

${ }^{15}$ Marek W, Mensing T, Fricke H, Baur X. Water-soluble cooling lubricants induce airway hyperresponsiveness in rabbits. Respiration 1998; 65: $143-150$

${ }^{16}$ Mensing T, Marek W, Fricke H, Wilhelm M, Baur X. Influence of nonwater and water soluble lubricant exposure on airway responsiveness in rabbits. Washington, D.C.: Ilsi Press, 2000: 398-401

${ }^{17}$ Kriebel D, Sama SR, Woskie S, Christiani D, Eisen EA, Hammond SK, Milton DK, Smith M, Virji MA. A field investigation of acute respiratory effects of metal working fluids. I. Effects of aerosol exposures. Am J Ind Med 1997; 31: $756-766$
${ }^{18}$ Kennedy SM, Greaves IA, Kriebel D, Eisen EA, Smith TJ, Woskie SR. Acute pulmonary responses among automobile workers exposed to aerosols of machining fluids. Am J Ind Med 1989; 15: 627-641

${ }^{19}$ Sprince NL, Thorne PS, Popendorf W, Zwerling C, Miller ER, DeKoster JA. Respiratory symptoms and lung function abnormalities among machine operators in automobile production. Am J Ind Med 1997; 31: $403-413$

20 Oxhoj H, Andreasen H, Meyer-Henius U. Respiratory symptoms and ventilatory lung function in machine shop workers exposed to coolant-lubricants. Eur J Respir Dis 1982; 118: 85-89

${ }^{21}$ Järvholm B, Bake B, Lavenius B, Thiringer G, Vokmann R. Respiratory symptoms and lung function in oil mist-exposed workers. J Occup Med 1982; 24: 473-479

${ }^{22}$ Massin N, Bohadana AB, Wild P, Goutet P, Kirstetter H, Toamain JP. Airway responsiveness, respiratory symptoms and exposure to soluble oil mist in mechanical workers. Occup Environ Med 1996; 53: $748-752$

${ }^{23}$ Robertson AS, Weir DC, Sherwood Burge P. Occupational asthma due to oil mists. Thorax 1988; 43: 200-205

${ }^{24}$ Hendy MS, Beattie BE, Burge PS. Occupational asthma due to an emulsified oil mist. Brit J Ind Med 1985; 42: 51 - 54

${ }^{25}$ Prelicz H, Morawa R, Greger G, Konofsky B, Petro W. Allergisches, berufsbedingte Asthma bronchiale durch Kühlschmiermittelaerosole. Arbeitsmed Sozialmed Präventivmed 1991; 26: 67-69

${ }^{26}$ Stork J, Labrot B, Mann H, Nöring R, Spallek M. Untersuchung zur Häufigkeit der chronischen Bronchitis bei Schneidöl-Exposition. Verh Dtsch Ges Arbeitsmed Stuttgart: Gentner Verlag, 1989: 383 - 386

27 Stork J, Spallek M, Labrot B, Mann H, Nöring R, Saake P. Akuter Einfluss einer Exposition gegenüber Aerosolen eines wassermischbaren Kühlschmierstoffs auf Messwerte der Lungenfunktion? Arbeitsmed Sozialmed Präventivmed 1990; 25: 372 - 374

${ }^{28}$ Kennedy SM, Chan Jeung M, Teschke K, Karlen B. Change in airway responsiveness among apprentices exposed to metal working fluids. Am J Respir Crit Care Med 1999; 159: 87-93

29 American Conference of Governmental Industrial Hygienists. TLVs and BEIS Cincinnati, USA, 2000

${ }^{30}$ Rosenmann KD, Reilly MJ, Kalinowski D. Work-related asthma and respiratory symptoms among workers exposed to metal-working fluids. Am J Ind Med 1997; 32: 325-331

31 Freemann A, Lockey J, Hawley P, Biddinger P, Trout D. Hypersensitivity pneumonitis in a machinist. Am J Ind Med 1998; 34: 387-392

32 Fox J, Anderson H, Moen T, Gruetzmacher G, Hanrahan L, Fink J. Metal working fluid-associated hypersensitivity pneumonitis: an outbreak investigation and case-control study. Am J Ind Med 1999; 35: 58 - 67

${ }^{33}$ Hodgson MJ, Bracker A, Yang C, Storey E, Jarvis BJ, Milton D, Lummus Z, Bernstein D, Cole S. Hypersensitivity pneumonitis in a metal-working environment. Am J Ind Med 2001; 39: 616-628

${ }^{34}$ Zacharisen MC, Kadambi AR, Schlueter DP, Kurup VP, Shack JB, Fox JL, Anderson HA, Fink JN. The spectrum of respiratory disease associated with exposure to metal working fluids. JOEM 1998; 40: 640-647

${ }^{35}$ Hörner D. Kühlschmierstoffe für die Minimalmengenschmierung. In: Umweltfreundlich Zerspanen. VDI Berichte 1339 Düsseldorf: VDI Verlag, 1997: 203-241

${ }^{36}$ Robins T, Seixas N, Franzblau A, Abrams L, Minick S, Burge H, Schork MA. Acute respiratory effects on workers exposed to metalworking fluid aerosols in an automotive transmission plant. Am J Ind Med 1997; 31: 510-524

37 Wild P, Ameille J. Bronchial reactivity in oil-mist exposed automobile workers revisited. Letter to the editor. Am J Ind Med 1997; 32: $421-422$

${ }^{38}$ Ameille J, Wild P, Choudat D, Ohl G, Vaucouleur JF, Chanut JC, Brochard P. Respiratory symptoms, ventilatory impairment, and bronchial reactivity in oil mist-exposed automobile workers. Am J Ind Med 1995; 27: $247-256$ 